\title{
Características Antropométricas de Adolescentes Pertenecientes a Distintas Escuelas Deportivas Formativas
}

\author{
Anthropometric Characteristics of Adolescents from Different Sports Training Schools

\begin{abstract}
Andrés Esteban Roberto Godoy-Cumillaf; Pablo Antonio Valdés-Badilla***; Noemí Salvador Soler ${ }^{* * *}$;
\end{abstract} \\ María Inés Carmona-López ${ }^{* * *}$ \& Juan José Fernández ${ }^{* * *}$
}

GODOY-CUMILLAF, A. E. R.; VALDÉS-BADILLA, P. A.; SALVADOR, S. N.; CARMONA-LÓPEZ, M. I. \& FERNÁNDEZ, J. J. Características antropométricas de adolescentes pertenecientes a distintas escuelas deportivas formativas. Int. J. Morphol., 33(3):1065$1070,2015$.

RESUMEN: La alta tasa de sedentarismo y obesidad reportada en Chile, ha propiciado la aparición de diversos programas deportivos para niños y adolescentes, que requieren evaluaciones fiables en torno a las características antropométricas por disciplina Deportiva. En este sentido, la presente investigación pretende por objetivo determinar las características antropométricas de adolescentes pertenecientes a distintas escuelas deportivas formativas (AD) de Temuco, Chile y su relación con parámetros deportivos ideales. El tipo de investigación contempla un diseño no experimental, descriptivo, transversal, con un enfoque cuantitativo. La muestra estuvo constituida por 65 sujetos (38 mujeres) con un promedio de edad de $15,9(2,5)$ años y un IMC de 22,8 (4) kg/m², quienes practicaban taekwondo $(n=4)$, racquetbol $(n=6)$, esgrima $(n=7)$, karate $(n=8)$, atletismo $(n=13)$, tenis $(n=6)$ y fútbol $(n=21)$. Los resultados indican que las AD mujeres promediaron un somatotipo de 4,9-4,3-1,5 (clasificadas como endomesomorfas), masa adiposa de 33,6\% y masa muscular de $39,7 \%$, mientras los AD varones para las mismas variables ostentan 3-4,9-2,3 (mesoendomorfos), 27,4\% y 43,7\%. En conclusión, los AD evaluados ostentan normopeso, su composición corporal exhibe una elevada masa adiposa incluso para parámetros normales, mientras que su somatotipo parece acercarse a los ideales deportivos, sin evidenciar una tendencia por disciplina deportiva. Se sugiere para futuras investigaciones estudiar otras variables, como los programas de entrenamiento, el rendimiento deportivo y los hábitos alimentarios, con la intención de obtener respuestas más satisfactorias respecto a los factores que influyen en la proyección de adolescentes al deporte competitivo.

PALABRAS CLAVE: Somatotipo; Composición corporal; Estado nutricional; Índice de masa corporal; Taekwondo; Racquetbol; Esgrima; Karate; Atletismo; Tenis y Fútbol.

\section{INTRODUCCIÓN}

Chile es uno de los países con mayor prevalencia de sedentarismo en el mundo, alcanzando al $88,6 \%$ de la población adulta (MINSAL, 2011), mientras que ostenta un $44 \%$ de sobrepeso y obesidad escolar (Agencia de Calidad de la Educación, 2013). Cifras preocupantes y que sugieren acciones preventivas desde edades tempranas, siendo la adolescencia una etapa sensible y de gran influencia en las conductas de vida futura (Ré, 2011).

Por otra parte, existe suficiente evidencia de los beneficios que producen la actividad física y el deporte sobre la salud de las personas (Hoehner et al., 2013; Zhao et al., 2014), las cuales impactan positivamente sobre factores de riesgo, como son: la obesidad, la resistencia a la insulina y otras en- fermedades no transmisibles propias de los tiempos actuales. Además, distintos autores (González et al., 2009; González \& González, 2010; Matveyev, 2004; Martin et al., 2001) coinciden en propiciar el desarrollo multilateral y de todas las cualidades físicas durante la práctica de deporte formativo, con la intención de fortalecer armónicamente la estructura corporal de niños y adolescentes a través de propuestas metodológicas atractivas y que despierten su interés duradero.

En este contexto en Chile, se han instaurado en los últimos años distintos programas gubernamentales que han posibilitado la concepción de talleres y escuelas orientadas al deporte formativo en todo el país (Gobierno de Chile, 2001), los que ostentan sistemas de evaluación variados o

* Departamento de Educación Física, Facultad de Educación, Universidad Autónoma de Chile, sede Temuco, Chile.

** Instituto de Actividad Física y Salud, Universidad Autónoma de Chile, Chile.

**** Instituto de Ciencias Biomédicas, Facultad de Ciencias de la Salud, Universidad Autónoma de Chile, sede Temuco, Chile. 
escasos, perjudicando el seguimiento de los beneficiarios como de su posible proyección al deporte competitivo. Por esta razón, las referencias antropométricas constituyen un parámetro fiable al momento de evaluar el biotipo deportivo de acuerdo a los estándares específicos de cada disciplina (Marfell-Jones et al., 2006; Ramos-Campo et al., 2014).

En este sentido, la presente investigación pretende por objetivo determinar las características antropométricas de adolescentes pertenecientes a distintas escuelas deportivas formativas de Temuco, Chile (AD) y su relación con parámetros deportivos ideales.

\section{MATERIAL Y MÉTODO}

El tipo de investigación contempla un diseño no experimental, descriptivo, transversal, con un enfoque cuantitativo.

La población estuvo constituida por todos los adolescentes pertenecientes a las escuelas deportivas formativas desarrolladas en un centro Polideportivo de la ciudad de Temuco, Chile ( $\mathrm{n}=88$ ). La muestra fue seleccionada bajo un criterio no probabilístico, que incluyó a $65(73,8 \%)$ sujetos, divididos en 38 mujeres y 27 varones, quienes practicaban taekwondo $(n=4)$, racquetbol $(n=6)$, esgrima $(n=7)$, karate $(n=8)$, atletismo $(n=13)$, tenis $(n=$ 6) y fútbol $(n=21)$. Los adolescentes entrenaban 3 veces por semana (60 a 90 minutos por sesión), con un promedio de 3 años de práctica, edad de $15,9(2,5)$ años, talla de $1,65(0,08)$ metros, peso de 59,7 $\pm 10,7$ kilos y un IMC de $22,8(4) \mathrm{kg} / \mathrm{m}^{2}$ (datos que pueden apreciarse en la Tabla I).

Respecto a los criterios de inclusión, se invitó a todos los adolescentes deportistas (AD) a participar de la investigación, debiendo cumplir con la firma de un asentimiento y consentimiento informado de los Padres que autoriza el uso de la información con fines científicos; excluyendo a quienes no asistieron, no firmaron el documento solicitado o no quisieron participar de las evaluaciones. El estudio fue aprobado por el Comité de Ética de la Universidad Autónoma de Chile y desarrollado siguiendo lo expuesto en la Declaración de Helsinki.

Todas las evaluaciones se realizaron entre los meses de octubre y noviembre del 2014, durante el periodo lectivo de las escuelas, ocupando un recinto cerrado y calefaccionado a una temperatura de $18{ }^{\circ} \mathrm{C}$ y un $50 \%$ de humedad relativa. Se obtuvieron todas las mediciones a través de evaluadores certificados con nivel II de la ISAK.
Para realizar las evaluaciones, se comenzó con el somatotipo, el cual se fraccionó según el método descrito por Heath \& Carter (1967). Las medidas fueron tomadas según el protocolo descrito por la ISAK (Marfell-Jones et al.). El peso corporal fue evaluado con balanza digital (Scale-tronix, USA), la estatura con estadiómetro (Seca modelo 220, Alemania), los diámetros con antropómetros (Rosscraft, Canadá), los perímetros con cinta métrica (Sanny, Brasil) y los pliegues cutáneos con cáliper (Harpenden, Inglaterra). Los instrumentos ostentan una precisión de $0,1 \mathrm{~kg}, 0,10 \mathrm{~cm}, 0,1 \mathrm{~mm}, 0,1 \mathrm{~mm}$ y $0,2 \mathrm{~mm}$, respectivamente. Además, se calculó el índice de masa corporal (IMC) para categorizar el estado nutricional de acuerdo a los criterios establecidos por la OMS (2000); y el índice cintura cadera (ICC) para valorar el riesgo cardiovascular (Remón et al., 2013).

Para la tabulación de los datos se utilizó el programa Microsoft Excel versión 7.0 y para el tratamiento estadístico el software Statgraphics 5.1. Con la prueba de Shapiro-Wilk se evaluó si las variables presentaban una distribución normal. Las variables que cumplían con dicha condición fueron descritas mediante la media aritmética y la desviación estándar (Media \pm SD), y la comparación entre ambos sexos fue realizada con la prueba tStudent. Las variables que no presentaron una distribución normal fueron descritas mediante la mediana y el rango interquartil (Me [rango]) y la comparación entre sexos fue realizada con la prueba de Mann-Whitney, ésta última realizada con el software Statistical Package for Social Science (SPSS) versión 20.0, considerando un nivel significativo de $\mathrm{p}<0,05$.

\section{RESULTADOS}

En la Tabla I se presenta la caracterización de los AD de las distintas escuelas formativas, distribuidos por género. Se observaron diferencias estadísticamente significativas entre mujeres y varones para las variables de talla, peso corporal, perímetro de cintura e índice cintura-cadera, siendo los varones quienes presentaron los valores más altos. En cuanto a la composición corporal de los $\mathrm{AD}$, se aprecian registros más altos de los varones respecto a las mujeres en masa muscular, masa residual y masa ósea, siendo estas diferencias estadísticamente significativas, mientras que las mujeres presentaron mayor porcentaje de masa grasa y masa epitelial, respectivamente.

La Tabla II expone los resultados para el somatotipo promedio de los $\mathrm{AD}$ distribuidos por disciplina deportiva, evidenciando los valores respecto al ideal deportivo según 
GODOY-CUMILLAF, A. E. R.; VALDÉS-BADILLA, P. A.; SALVADOR, S. N.; CARMONA-LÓPEZ, M. I. \& FERNÁNDEZ, J. J. Características antropométricas de adolescentes pertenecientes a distintas escuelas deportivas formativas. Int. J. Morphol., 33(3):1065-1070, 2015.

Tabla I. Características antropométricas de los adolescentes deportistas pertenecientes a distintas escuelas deportivas formativas.

\begin{tabular}{lccccc}
\hline \multirow{2}{*}{ Datos } & \multirow{2}{*}{ Mínimo } & Mujeres $(\mathbf{n}=\mathbf{3 8})$ & Varones $(\mathbf{n}=\mathbf{2 7})$ & \multirow{2}{*}{ Máximo } & \multirow{2}{*}{ Valor p } \\
\cline { 3 - 4 } & & Media $\pm \mathbf{D E}$ & Media $\pm \mathbf{D E}$ & & \\
\hline Edad (años) & 11,1 & $16,2 \pm 2,3$ & $15,6 \pm 2,7$ & 20,8 & 0,001 \\
Talla (m)* & 1,11 & $1,6 \pm 0,08$ & $1,7 \pm 0,08$ & 1,79 & 0,002 \\
Peso corporal (kg)* & 30 & $56,6 \pm 12,2$ & $62,8 \pm 9,1$ & 84 & 0,822 \\
IMC (Kg/m²) & 18,4 & $22,5 \pm 4,8$ & $23 \pm 3,2$ & 26,4 & 0,001 \\
PC (cm)* & 57,7 & $71,2 \pm 7,5$ & $75,8 \pm 7,2$ & 96,5 & 0,812 \\
PCA (cm) & 73,2 & $94,4 \pm 9,1$ & $92,3 \pm 6,0$ & 115,3 & 0,882 \\
ICC* & 0,75 & $0,75 \pm 0,08$ & $0,81 \pm 0,07$ & 0,84 & 0,002 \\
Masa Adiposa (\%)* & 21,5 & $33,6 \pm 3,3$ & $27,4 \pm 3,9$ & 43 & 0,421 \\
Masa Muscular (\%)* & 34,6 & $39,7 \pm 2,3$ & $43,7 \pm 3,6$ & 48,8 & 0,091 \\
Masa Residual (\%)* & 7,9 & $9,75 \pm 1,1$ & $10,9 \pm 1,6$ & 15,3 & 0,022 \\
Masa Ósea (\%)* & 8,8 & $11,3 \pm 1,0$ & $12 \pm 1,1$ & 14 & 0,743 \\
Masa epitelial (\%) & 4,6 & $5,7 \pm 0,9$ & $5,4 \pm 0,7$ & 7,5 & 0,003 \\
Endomorfia* & 1,7 & $4,9 \pm 2,4$ & $3 \pm 1,6$ & 7,6 & 0,011 \\
Mesomorfía* & 2,2 & $4,3 \pm 1,0$ & $4,9 \pm 1,1$ & 6,9 & 0,262 \\
Ectomorfía* & 0,1 & $1,5 \pm 1,3$ & $2,3 \pm 1,8$ & 2,2 & 0,022 \\
\hline
\end{tabular}

$\mathrm{IMC}=$ índice de masa corporal $; \mathrm{PC}=$ perímetro de cintura; $\mathrm{PCA}=$ perímetro de cadera; $\mathrm{ICC}=$ índice cintura cadera; $*=\mathrm{Diferencias}$ significativas ( $\mathrm{p}$-valor $<0,05$ ).

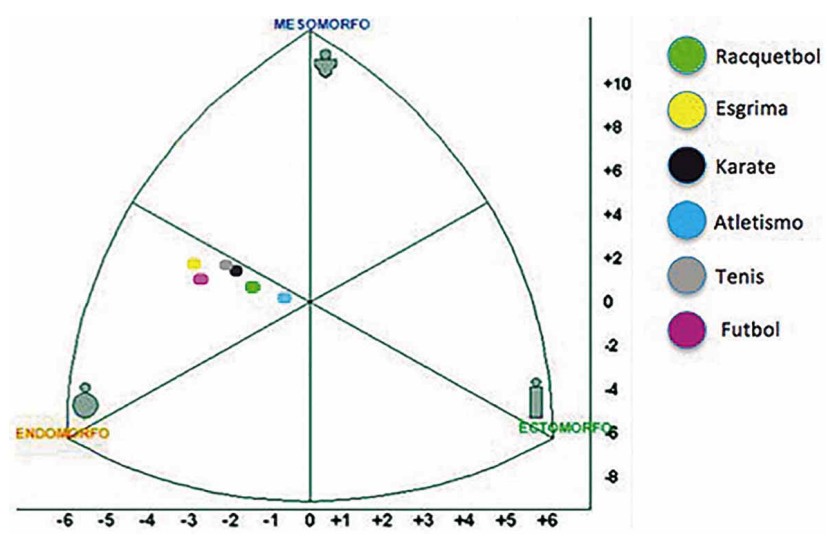

Fig. 1. Ubicación promedio en la somatocarta de las mujeres adolescentes deportistas pertenecientes a distintas escuelas deportivas formativas.

lo plantean Lentini et al. (2004) y Rodríguez et al. (2014), de este modo se puede observar que los AD de taekwondo, racquetbol mujeres, karate varones, atletismo mujeres y varones, y tenis varones se encuentran cercanos a las medidas ideales. Mientras que se aprecian características somatotípicas distantes de los AD de racquetbol varones, esgrima mujeres y varones, karate mujeres, tenis mujeres y fútbol mujeres (Lentini et al.; Rodríguez et al.).

La Figura 1 presenta la ubicación somatotípica promedio de las AD mujeres de las distintas disciplinas deportivas de la muestra, mientras que la Figura 2 expone la distribución somatotípica promedio de los $\mathrm{AD}$ varones.

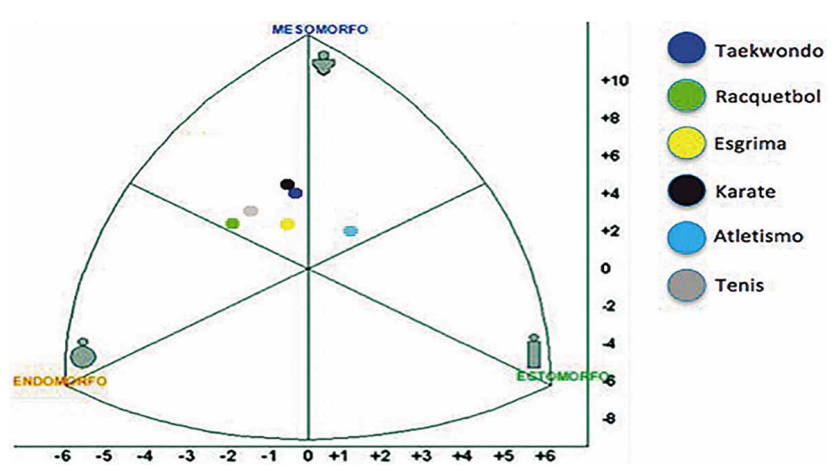

Fig. 2. Ubicación promedio en la somatocarta de los varones adolescentes deportistas pertenecientes a distintas escuelas deportivas formativas.

\section{DISCUSIÓN}

Los valores encontrados para el estado nutricional promedio de los AD evaluados, medido a través del IMC, alcanzaron $22,5 \mathrm{Kg} / \mathrm{m}^{2}$ para los varones, mientras que las mujeres registraron $23 \mathrm{Kg} / \mathrm{m}^{2}$. Datos que sitúan a los $\mathrm{AD}$ con normopeso, similar a lo reportado para deportistas de alto rendimiento de diferentes disciplinas (Lentini et al.; Rodríguez et al.). No obstante y según lo plantean Marfell-Jones et al., el IMC no aporta confiabilidad en las mediciones para deportistas, debido a que no diferencia entre masa adiposa y masa muscular. En este contexto, la composición corporal otorga 
Tabla II. Distribución somatotípica de los adolescentes deportistas pertenecientes a distintas escuelas deportivas formativas y valores somatotípicos ideales para deportistas élite.

\begin{tabular}{|c|c|c|c|c|}
\hline \multirow[t]{2}{*}{ Disciplina } & & \multicolumn{3}{|c|}{ Somatotipo } \\
\hline & & Endomorfía & Mesomorfía & Ectomorfía \\
\hline \multirow[t]{2}{*}{ Taekwondo } & Varones $(n=4)$ & $2,7 \pm 0,9$ & $5 \pm 0,8$ & $2,3 \pm 0,9$ \\
\hline & Ideal (V) & 2,0 & 4,7 & 2,9 \\
\hline \multirow[t]{4}{*}{ Racquetbol } & Mujeres $(n=1)$ & $4,9 \pm 2,8$ & $4,3 \pm 2,3$ & $2,8 \pm 3,0$ \\
\hline & Ideal (M) & 5,0 & 4,8 & 2,0 \\
\hline & Varones $(n=5)$ & $4,4 \pm 1,8$ & $5 \pm 1,4$ & $2,5 \pm 1,9$ \\
\hline & Ideal $(\mathrm{V})$ & 1,9 & 4,9 & 2,4 \\
\hline \multirow[t]{4}{*}{ Esgrima } & Mujeres $(n=3)$ & $5,1 \pm 1,4$ & $4,6 \pm 0,6$ & $1,6 \pm 1,0$ \\
\hline & Ideal (M) & 3,9 & 3,4 & 2,4 \\
\hline & Varones $(n=4)$ & $3,1 \pm 0,7$ & $4,5 \pm 0,5$ & $2,6 \pm 0,8$ \\
\hline & Ideal $(\mathrm{V})$ & 3,6 & 6,2 & 1,3 \\
\hline \multirow[t]{4}{*}{ Karate } & Mujeres $(n=4)$ & $4,3 \pm 0,8$ & $4,1 \pm 0,8$ & $2,2 \pm 1,4$ \\
\hline & Ideal (M) & 3,0 & 5,8 & 1,5 \\
\hline & Varones $(n=4)$ & $2,9 \pm 0,9$ & $5,8 \pm 1,1$ & $2,4 \pm 1,4$ \\
\hline & Ideal (V) & 3,1 & 5,4 & 1,8 \\
\hline \multirow{4}{*}{$\begin{array}{l}\text { Atletismo } \\
\text { (velocidad) }\end{array}$} & Mujeres $(n=8)$ & $3,5 \pm 0,9$ & $3,4 \pm 0,7$ & $2,9 \pm 0,9$ \\
\hline & Ideal (M) & 2,5 & 3,4 & 3,2 \\
\hline & Varones $(n=5)$ & $2,4 \pm 0,4$ & $4,2 \pm 1,2$ & $3,4 \pm 0,7$ \\
\hline & Ideal $(\mathrm{V})$ & 1,9 & 5,2 & 2,5 \\
\hline \multirow[t]{4}{*}{ Tenis } & Mujeres $(n=1)$ & 4,8 & 4,5 & 1,5 \\
\hline & Ideal (M) & 3,5 & 3,6 & 2,7 \\
\hline & Varones $(n=5)$ & $3,8 \pm 1,4$ & $5,3 \pm 0,9$ & $1,6 \pm 0,7$ \\
\hline & Ideal (V) & 2,3 & 4,4 & 2,9 \\
\hline \multirow[t]{2}{*}{ Fútbol } & Mujeres $(n=21)$ & $5,9 \pm 1,0$ & $4,9 \pm 0,9$ & $2,2 \pm 4,8$ \\
\hline & Ideal (M) & 4,2 & 3,77 & 2,04 \\
\hline
\end{tabular}

$\mathrm{V}=$ varones; $\mathrm{M}=$ mujeres. Valores somatotípicos ideales para deportistas adultos élite de acuerdo a los datos obtenidos de: Rodríguez et al. (2014) y Lentini et al. (2010). que indican una clasificación de mesomorfos balanceados. Por su parte, la $\mathrm{AD}$ mujer de racquetbol, alcanzó una categorización de endomesomorfa $(4,9$ - 4,3 - 2,8), manifestando mayor cantidad de masa adiposa, luego de masa muscular, siendo estos valores similares a los reportados en argentinas de la misma especialidad, que se categorizaron como endomesormofas balanceadas $(5-4,8-2)$ (Lentini et al.).

En relación a los $\mathrm{AD}$ varones practicantes de esgrima, su somatotipo alcanzó la clasificación de mesoendomorfos $(3,1-4,5-2,6)$, antecedentes similares a los señalados por Rodríguez et al., quienes encontraron en esgrimistas chilenos élite, una categorización de mesoendomorfos $(3,6-6,2-1,3)$. A su vez, las AD mujeres de esgrima obtuvieron una clasificación de endomesomorfas balanceadas $(5,1-4,6-1,6)$, similar a la reportada por Rodríguez et al., para esgrimistas chilenas élite que alcanzaron un somatotipo endomesomorfo balanceado $(3,9-3,4-2,4)$.

Respecto a los AD varones de información más precisa de la constitución física de los $\mathrm{AD}$, situándolos en categoría elevado para masa adiposa tanto mujeres $(33,6 \%)$ como varones $(27,4 \%)$, esto de acuerdo a tablas normativas para adolescentes y adultos normales (Marfell-Jones et al.).

Al indagar en torno a las características somatotípicas de los AD distribuidos por disciplina deportiva, se puede senalar que los $\mathrm{AD}$ varones practicantes de taekwondo presentaron una clasificación somatotípica mesomórfica balanceada $(2,7-5-2,3)$, lo que indica predominio del tejido músculo esquelético, no exhibiendo mayores diferencias entre los componentes endomórficos y ectomórficos. Al respecto, Betancourt et al. (2009) reportaron en atletas cubanos de taekwondo élite, un somatotipo mesoectomorfo (1,8 - 4,4 $3,5)$, exhibiendo predominancia del componente mesomórfico, similar al encontrado en los AD de taekwondo.

En cuanto a los AD varones de racquetbol, se observó un somatotipo mesoendomorfo $(4,4-5-2,5)$, que señala dominancia de la masa muscular, seguida de la masa adiposa, datos distintos a los encontrados por Lentini et al., en atletas argentinos de la especialidad de racquetbol $(1,9-4,9-2,4)$ karate, su clasificación somatotípica se situó en mesoendomorfos $(2,9-5,8-2,4)$, situación algo distinta a la reportada por Sánchez-Puccini et al. (2014), quienes evaluaron a karatekas élite de Colombia, encontrando un categorización promedio de mesoendomorfos balanceados $(4,0$ $-4,2$ - 2,1). Por su parte, las AD mujeres de karate, alcanzaron un somatotipo de endomesomorfas balanceadas $(4,3-4,1$ $-2,2)$, clasificación distinta a la encontrada por Lentini et al., en karatekas mujeres adultas élite que se ubicaron como mesoendomorfas balanceadas $(3,7-3,9-2)$.

En referencia a los AD varones de atletismo de velocidad, estos ostentan un somatotipo mesoectomorfo (2,4 - 4,2 $3,4)$, escenario distinto al señalado por Avella \& Medellín (2013) para varones adultos élite de atletismo, encontrando una clasificación de mesoendomorfos $(2,6-4-2)$. Mientras que las $\mathrm{AD}$ mujeres de atletismo alcanzan una clasificación de endomesomorfas balanceados $(3,5-3,4-2,9)$, similar a lo reportado por Avella \& Medellín, en atletas adultas élite (3,2 $3,1-3)$.

En cuanto a los AD varones practicantes de tenis, su clasificación somatotípica se enmarca como mesoendomorfos 
$(3,8-5,3-1,6)$, mientras que tenistas élite argentinos (Lentini et al.), se ubican como mesoectomorfos $(2,3-4,4$ $-2,9)$. Las AD mujeres de tenis, obtienen un categorización de endomesomorfas balanceadas $(4,8-4,5-1,5)$, dato distinto al alcanzado por tenistas argentinas (Lentini et al.), quienes ostentan un somatotipo de mesoendomorfas balanceadas $(3,5-3,6-2,7)$.

En relación a las $\mathrm{AD}$ de fútbol femenino, su somatotipo promedio fue de endomesomorfas $(5,9-4,9-$ $2,2)$, siendo similar a lo encontrado por Bahamondes et al. (2012) para futbolistas mujeres sub-17 de nivel sudamericano $(4,2-3,8-2)$.

Es importante destacar que independiente el deporte los $\mathrm{AD}$ varones ostentan una predominancia del componente mesomórfico (masa muscular) en su constitución física, mientras que en las $\mathrm{AD}$ mujeres prima la endomorfía (masa adiposa). Esta situación concuerda con lo expuesto por Marfell-Jones et al., respecto a la predominancia de la masa adiposa en las mujeres, que a su vez, confirma Tanner (1978) cuando indica que la masa muscular es mayor en varones, siendo complementado por López \& Fernandez (2006) cuando señalan que, la pubertad produce un aumento de la testosterona en los varones que favorece el desarrollo de masa muscular.

Las diferencias existentes entre los registros encontrados en los AD y el valor somatotípico ideal en cada una de las disciplinas deportivas analizadas, se relacionan en primer término con la edad de los sujetos, quienes promedian 15,9 años y de acuerdo a lo expuesto por López \& Fernández, los adolescentes aún no alcanzan el mayor potencial de su masa muscular, que se obtiene entre los 20 y los 30 años.

Otro factor que influye en los resultados obtenidos, corresponde al volumen de entrenamiento de los AD que se encuentra entre 3 y 5 horas semanales, mientras que los deportistas de referencia pertenecen a selecciones nacionales y su carga de entrenamiento semanal se sitúa entre 12 y 20 h. En este sentido, diferentes autores señalan que la práctica regular de ejercicio físico produce efectos hipertróficos en la musculatura así como disminución de la masa adiposa (Martins \& Rodríguez Dos Santos, 2004; López \& Fernández).

Por otra parte, la alimentación jugaría un papel importante en las características antropométricas de los $\mathrm{AD}$, ya que como lo plantean Heath \& Carter, el somatotipo puede ser modificado a lo largo de la vida y de acuerdo a Holway \& Guerci (2012) los hábitos alimentarios influyen notablemente en la composición corporal y el perfil somatotípico de los sujetos.
Es importante considerar que muchos autores (González et al.; González \& González; Matveyev; Martin et al.) indican que el rendimiento deportivo es multifactorial, por tanto, entre más variables sean controladas durante el entrenamiento y la competencia mayor será la probabilidad de obtener resultados satisfactorios. En este contexto, estudiar las características antropométricas de los deportistas adolescentes sirve de insumo a entrenadores, profesores y técnicos para proponer planificaciones más precisas y que se encuentren en concordancia con los aspectos particulares de cada disciplina deportiva.

\section{CONCLUSIÓN}

Si bien los AD evaluados ostentan normopeso, su composición corporal exhibe una elevada masa adiposa incluso para parámetros normales, mientras que su somatotipo parece acercarse a los ideales deportivos, sin evidenciar una tendencia por disciplina deportiva. Se sugiere para futuras investigaciones estudiar otras variables, como los programas de entrenamiento, el rendimiento deportivo y los hábitos alimentarios, con la intención de obtener respuestas más satisfactorias respecto a los factores que influyen en la proyección de adolescentes al deporte competitivo.

AGRADECIMIENTOS. A Erwin Lincoqueo por su disposición en la logística del estudio y a todos los deportistas que participaron de las evaluaciones.

GODOY-CUMILLAF, A. E. R.; VALDÉS-BADILLA, P. A.; SALVADOR, S. N.; CARMONA-LÓPEZ, M. I. \& FERNÁNDEZ, J. J. Anthropometric characteristics of adolescents from different sports training schools. Int. J. Morphol., 33(3):1065-1070, 2015.

SUMMARY: The high rate of physical inactivity and obesity reported in Chile, has given rise to various sports programs for children and adolescents who require reliable assessments of anthropometric characteristics for sports. In this sense, this research aims to determine the anthropometric characteristics of adolescents belonging to different training sports schools (AD) of Temuco, Chile and its relationship with ideal athletic parameters. The research provides a non-experimental, descriptive, transversal, with a quantitative approach to design. The sample consisted of 65 subjects ( 38 women) with a mean age of 15.9 (2.5) years and a BMI of 22.8 (4) $\mathrm{kg} / \mathrm{m}^{2}$, who practiced taekwondo ( $\mathrm{n}=$ $4)$, racquetball $(n=6)$, fencing $(n=7)$, karate $(n=8)$, athletics $(n=13)$, tennis $(n=6)$ andsoccer $(n=21)$. The results indicated that $A D$ women were classified as endo-mesomorphic $(4.9-4.3-1.5)$ with a mean percentage of $33.6 \%$ fat mass and $39.7 \%$ muscle mass, while the $\mathrm{AD}$ males were classified as meso-endomorphic $(3-4.9-2.3)$ and they presented a $27.4 \%$ of fat mass and a $43.7 \%$ of muscle mass. In conclusion, although the $\mathrm{AD}$ evaluated showed normal weight, their body composition exhibits a high percentage of fat mass, even in 
comparison with normal parameters. Nevertheless, its somatotype was closer to the kineanthropometric profile ideal for sports, without showing any trend by sport. Further studies will be required to understand the role of training programs, athletic performance and eating habits in the optimization of sports performance.

KEY WORDS: Somatotype; Body composition; Nutritional status; Body mass index; Taekwondo, Racquetball; Fencing; Karate; Athletics; Tennis and soccer.

\section{REFERENCIAS BIBLIOGRÁFICAS}

Agencia de Calidad de la Educación. Informe de Resultados SIMCE Educación Física $8 .^{\circ}$ Básico 2012 para Docentes y Directivos. Santiago de Chile, Gobierno de Chile, 2013. Disponible en: http:// www.agenciaeducacion.cl/wp-content/files_mf/ ir_educacion_fisica_2012.pdf

Avella, R. E. \& Medellín, J. P. Perfil dermatoglífico y somatotípico de atletas de la selección Colombia de atletismo (velocidad) participante en los Juegos Panamericanos de Guadalajara, 2011. Rev. U. D. C. A. Act. Div. Cient., 16(1):17-25, 2013.

Bahamondes, A. C.; Cifuentes, C. B. M.; Padilla, E. L. \& Berral de la Rosa, F. J. Body Composition and Somatotype in Women's Football. South American Championship Sub-17. Int. J. Morphol., 30(2):45060, 2012 .

Betancourt, L. H.; Aréchiga, V. J. \& Carvajal, V. W. Estimación antropológica de la forma corporal de atletas elites cubanos de deportes olímpicos de combate. Antropo, 19:23-32, 2009.

Gobierno de Chile. Ley $N^{o}$ 20737. Ley del deporte. Santiago de Chile, Gobierno de Chile, 2001.

González, V. S.; Garcia, L. L. M.; Contreras, J. O. R. \& Sánchez, M. M. D. El concepto de iniciación deportiva en la actualidad. Retos. Nuevas tend. Educ. Fis. Deporte Recreación, 15(1):14-20, 2009.

González, C. A. M. \& González, C. C. H. Educación física desde la corporeidad y la motricidad. Hacia Promoc. Salud, 15(2):173-87, 2010.

Heath, B. H. \& Carter, J. E. A modified somatotype method. Am. J. Phys. Anthropol., 27(1):57-74, 1967.

Hoehner, C. M.; Ribeiro, I. C.; Parra, D. C.; Reis, R. S.; Azevedo, M. R.; Hino, A. A.; Soares, J.; Hallal, P. C.; Simões, E. J. \& Brownson, R. C. Physical activity interventions in Latin America: expanding and classifying the evidence. Am. J. Prev. Med., 44(3):e31-40, 2013.

Holway, F. E. \& Guerci, G. Capacidad predictiva de los parámetros antropométricos y de maduración sobre el rendimiento de adolescentes noveles en remo-ergómetro. Apunts Med. Esport, 47(175):99-104, 2012.

Lentini, N. A.; Gris, G. M.; Cardey, M. L.; Aquilino, G. \& Dolce, P. A. Estudio somatotípico en deportistas de alto rendimiento de Argentina. Arch. Med. Deporte, 21(104):497-509, 2004.

López, C. J. \& Fernández, V. A. Fisiología del ejercicio. $3^{\text {a }}$ ed. Madrid, Médica Panamericana, 2006.
Marfell-Jones, M.; Olds, T.; Stewart, A. \& Carter, L. International Standards for Anthropometric Assessment. Potchefstroom, International Society for the Advancement of Kinanthropometry (ISAK), 2006.

Martins, F. R. \& Rodríguez Dos Santos, J. A. Atividade física de lazer, alimentação y composição corporal. Rev. Bras. Educ. Fís. Esporte (São Paulo), 18(2):159-67, 2004.

Martin, D.; Carl, K. \& Lehnertz, K. Manual de metodología del entrenamiento deportivo. Barcelona, Paidotribo, 2001. pp.337-67.

Matveyev, L. P. El proceso del entrenamiento deportivo. Buenos Aires, Stadium S. R. L., 2004.

Ministerio de Salud (MINSAL). Encuesta Nacional de Salud ENS Chile 2009-2010. Santiago de Chile, Gobierno de Chile, 2011. Disponible en: http://web.minsal.cl/portal/url/item/ bcb03d7bc28b64dfe040010165012d23.pdf

Ramos-Campo, D. J.; Martínez Sánchez, F.; Esteban García, P.; Rubio Arias, J. A.; Bores Cerezal, A.; Clemente-Suarez, V. J. \& Jiménez Díaz, J. F. Body composition features in different playing position of professional team indoor players: basketball, handball and futsal. Int. J. Morphol., 32(4):1316-24, 2014.

Ré, A. H. N. Crescimento, maturação e desenvolvimento na infância e adolescência: Implicações para o esporte. Motricidade, 7(3):55-67, 2011.

Remón, P. I.; González, S. O. C. \& Arpa, G. A. El índice cintura-talla como variable de acumulación de grasa para valorar riesgo cardiovascular. Rev. Cub. Med. Mil., 42(4):444-50, 2013.

Rodríguez, P. X.; Castillo, V. O; Tejo, C. J. \& Rozowski, N. J. Somatotipo de los deportistas de alto rendimiento de Santiago, Chile. Rev. Chil. Nutr., 41(1):29-39, 2014.

Sánchez-Puccini, M. B.; Argothy-Bucheli, R. E.; Meneses-Echávez, J. F.; López-Albán, C. A. \& Ramírez-Vélez, R. Anthropometric and Physical Fitness Characterization of Male Elite Karate Athletes. Int. J. Morphol., 32(3):1026-31, 2014.

Tanner, J. M. Foetus Into Man, Physical Growth from Conception to Maturity. London, Open Books Pub., 1978.

World Health Organization (WHO). Obesity: preventing and managing the global epidemic. Report of a WHO consultation. World Health Organ. Tech. Rep. Ser., 894:1-253, 2000.

Zhao, G.; Li, C.; Ford, E. S.; Fulton, J. E.; Carlson, S. A.; Okoro, C. A.; Wen, X. J. \& Balluz, L. S. Leisure-time aerobic physical activity, muscle-strengthening activity and mortality risks among US adults: the NHANES linked mortality study. Br. J. Sports Med., 48(3):244-9, 2014.
Dirección para Correspondencia:
María Ines Carmona López
Universidad Autónoma de Chile
Av. Alemania 01090
Temuco
CHILE

Email: inescl65@gmail.com

Recibido : 28-05-2015

Aceptado: 13-07-2015 